\title{
Incentive Aspects of the Standardization of baseline in the Project based mechanisms in the international environmental cooperation
}

\author{
$\underline{\text { H. Imai }}^{\text {a }}$, J. Akita ${ }^{\mathrm{b}}$ and H. Niizawa ${ }^{\mathrm{c}}$ \\ ${ }^{a}$ KIER, Kyoto University, \\ ${ }^{b}$ Department of Eoconomics, Graduate School, Tohoku University \\ ${ }^{c}$ Faculty of Economics, University of Hyogo \\ Email: imai@kier.kyoto-u.ac.jp
}

\begin{abstract}
CDM was the major source of supply of emission traded at EU-ETC, although there were several criticisms against it. We examine a possible standardization of baseline setting method as discussed in the CDM reform at KP of UNFCCC and its CDM-EB in order to simplify the procedure and mitigate the costs born by the applicant, which was blamed for one major source of eclipse of CDM projects in the LDC.. Especially we tried to examine the role of transaction costs and self-selection under such baseline setting method. Combining several assumptions, we show that various problems could come out, and we tend to favor resolving the issue of transaction costs by itself rather than resolution via the standardization of the baseline setting methods.
\end{abstract}

First, we set out a simple model with a fixed output level to introduce baseline setting corresponding to the current practice with perfect information, (which of course is an idealization). Compared to this is a standardized baseline setting method where at the certain level, baseline emission level of GHG (in terms of emission factor). We take the case of greenfield project due to increased demand in an industry, so that even without a registration as a CDM project and hence no credits revenue some project would take place with possibly more emissions.

Then introducing uncertainty into some parameters, we examine the set of potential projects attracted to CDM under each baseline setting method with transaction costs explicitly accounted for as a component of fixed costs. As an immediate and obvious conclusion, due to the alleviation of the transaction costs, smaller projects would be induced to apply for the CDM. However, because of our simplified setup where true baseline level is not accompanied with an increased production cost, no over-crediting takes place.

Next we consider a particular assumption due to Fischer that emission level after the project and the true baseline level are proportional. Under this assumption, it is possible that quite different types of projects are induced to apply for CDM, and therefore there is a possibility of over-crediting. Introducing the possibility of scale parameter and the parameter representing a true baseline emission level, we observe that several possibilities could coexist.

Finally we account for the proposal made by CDM-EB with some warning based on the observations made above. Then we conclude that even though the CDM itself may be doomed to fade out, the issue of correct measurement of emission reduction, and to that effect any activity for the public good, is the major issue in the international negotiation together with several new mechanisms proposed, and so the results obtained in this exercise here could be of certain values for the future argument in the international environmental policy, especially of the economic mehanisms.

Keywords: $\quad C D M$, baseline-credit 


\section{INTRODUCTION}

Clean Development Mechanism (CDM) is an economic mechanism adopted by Kyoto Protocol (KP) of UNFCCC (United Nations Framework Convention on Climate Change). This mechanism allows GHG (greenhouse effect gases) emission reduction projects carried out inside non-Annex I (those nations which have no obligation to reduce emissions) countries to be registered and certified amount of emission reduction can be traded at the emission trading which is also a mechanism adopted in KP. In fact during the first commitment period (2008-2012) of KP, it was the major source of supply at the EU-ETS (emission trading system) which is the largest market for emission trading. After 2013, both supply and market shrink and CDM as well as emission trading scheme is almost extinct.

Both alternatives to and reform of CDM are discussed. Here we take up a particular option called standardization of baseline setting methods for CDM projects and examine its possible functioning from the viewpoint of economic incentives. Since CDM is a baseline-credit scheme, it is important to set a baseline emission level for the emission reduction project, that represents the emission level if it were not for CDM. Several methods are created and listed as the approved methodologies by CDM EB (executive board), but many blamed it as very complicate and demanding procedure consisting of transaction costs preventing small projects or projects planned in poor countries from participating. At the same time, environmentally concerned parties worried about maintaining quality of credits so that they represent real reduction (cf. Wara and Victor (2008)).

Although CDM are not prospering in the second commitment period with fewer countries joining KP, there are several new mechanisms proposed to replace or complement current mechanisms. And in the discussion, how to measure the real emission reduction remains to be a major issue in the international environmental negotiation. In this sense, the studies of this mechanism may give us insight which would be applicable to many other schemes in the international frameworks.

\section{BASELINE SETTING AND STANDARDIZATION}

Given a project which would reduce GHG emission from a certain activity, one could apply it for CDM. For that, one needs to select a baseline setting methodologies listed by CDM office (mostly identified with the CDM EB (executive board)), and then a methodology specify what data to collect and the formula to compute the credits. However, in this process, required data could be difficult to gather and understanding of the methodology itself could be a barrier too. In particular, additionality test which asks the proponent to prove that the project would not be carried out without CDM is often tough, including calculation of internal rate of return, etc. There are cnsultants and DOE (designated operational entity) to assist, but they are costly.

Thus without damaging environmental integrity, simplification of the procedure for CDM is called for. And standardization is one keyword. Already CDM EB has examined some scheme and some has investigated its problems (cf. Hayashi and Michelowa (2013)). In this paper, we try to examine this problem from a broader perspectives.

\section{FRAMEWORK OF ANALYSIS}

\subsection{Notation}

With some generality, we define a project by

(a, c, b, e, c', c”, e', e”, K, F, F', F”, R, R', R')

where

a: scale of the project

c: costs per output exclusive of credits revenue

$\mathrm{b}$ : individualized baseline emission level (per output)

e: projection emission level (per output)

$\mathrm{K}$ : transaction costs of CDM

$\mathrm{F}$ : investment costs 
Imai, Akita, and Niizawa, standardized baseline

$\mathrm{R}$ : profits under CDM with individualized baseline

Variables with ' standing for these parameters when CDM under standardized baseline

Variables with " standing for these parameters when no CDM

(We assume that the same output is produced if it were not for CDM with a different technology represented by parameters with ".)

Note that the difference between c and c' is generated by the difference in CDM credits revenue alone. Let $d$ stand for the costs other than credits revenue (which are negative in magnitude). Our argument requires that $\mathrm{b}>\mathrm{e}$ and $\mathrm{e}$ ">e . Distinction of $\mathrm{F}$ and $\mathrm{F}$ " and $\mathrm{ac}$, and $\mathrm{ac}$ " is meant to capture the difference between scale dependent costs and independent costs, but since we discuss static relationship only, we may do away with $\mathrm{F}$ and F".

First, we assume that the price of the output is set at 1 . In this case, profits are given by

$\mathrm{R} "=\mathrm{a}(1-\mathrm{c} ")-\mathrm{F} " \quad$ without CDM

$\mathrm{R}=\mathrm{a}(1-\mathrm{c})-\mathrm{K}-\mathrm{F} \quad \mathrm{CDM}$ with individualized baseline

$\mathrm{R}$ '=a(1-c')-K'-F CDM with standardized baseline

Informational assumption in the main part of our analysis is that supposedly the project emission ae is measured correctly after the fact, (possibly with some costs). q is commonly known. Other variables are possibly private information, and we consider different cases where different set of variables are unknown with possibly some additional relationship among variables.

\subsection{Incentive for CDM}

Under the individualized baseline, the project satisfies the additionality requirement for the CDM with an individualized baseline is

$$
\mathrm{a}(1-\mathrm{c} ")-\mathrm{F} ">\mathrm{a}(1-\mathrm{d})-\mathrm{F}
$$

For it to be viable

$$
\mathrm{a}(1-\mathrm{c} ")-\mathrm{F} "<\mathrm{a}(1-\mathrm{d})-\mathrm{K}-\mathrm{F}+\mathrm{a}(\mathrm{b}-\mathrm{e}) \mathrm{q}
$$

where $\mathrm{q}$ is the emission price, must hold. We assume c">d-q(b-e). Note that the additionality constraint is met simultaneously under this idealized situation. When $\mathrm{F}=\mathrm{F}$ ", this condition becomes $\mathrm{K}+\mathrm{ad}-\mathrm{aq}(\mathrm{b}-$ e) $<$ ac" $<$ ad.

Let b' be the standardized baseline and d' be the standardized additionarity level which is less than or equal to $b^{\prime}$.

Under CDM with standardized baseline, these conditions are

$$
\mathrm{a}(1-\mathrm{c} ")-\mathrm{F} "<\mathrm{a}(1-\mathrm{d})-\mathrm{K} \text { '-F+a(b'-e)q }
$$

with additionality condition is met if $\mathrm{e}<\mathrm{d}$ ', which we assume to hold here.

To our knowledge, it has not been stressed much but the transaction costs eliminate certain projects which could have been qualified as CDM projects, although it is quite obvious, and provided that $\mathrm{K}^{\text {' }}<\mathrm{K}$, given $\mathrm{b}=\mathrm{b}$ ', the viable projects would increase if standardized baseline is employed. Let us adapt a simplifying assumption that $\mathrm{K}^{\prime}=0<\mathrm{K}$.

\subsection{Information and self-selection}

Suppose that a is distributed with a distribution function G(a). For the greatest value of a such that $G(a)=0$ holds, we assume that $\mathrm{R}$ " $>0$. Also assume that $\mathrm{F} "=0$ and hence additionality condition is automatically satisfied.

The size at which a CDM project with an individualized baseline breaks even with no CDM, is

$a(1)=(K+F) /(c "+(b-e) q-d)$, 
and similarly for CDM with a standardized baseline

$\mathrm{a}(2)=\mathrm{F} /(\mathrm{c} "+(\mathrm{b}-\mathrm{e}) \mathrm{q}-\mathrm{d})$

and hence by $\operatorname{Prob}(\mathrm{a}(2)<\mathrm{a}<\mathrm{a}(1))=\mathrm{G}(\mathrm{a}(1))-\mathrm{G}(\mathrm{a}(2))$, the chance of projects being viable increases by standardization of the baseline. Note that the only source is the transaction costs involving the procedure of CDM. However, from the viewpoint of the credit accounting, apparently over-crediting as a whole takes place, which is in a stark contrast with the case where variation in BAU emission is the main concern and transaction costs are not taken into consideration. This observation suggests that the matter is not only a balance of the credit accounting but also in the alleviation of transaction costs which may undermine the security of the emission reduction as a good.

In order to evaluate the overall effect, one must consider

$-\mathrm{q}$ \{change in credits $\}+$ (net change in profits) - (loss of exact measurement)

Where the first term represents the loss of emission reduction in the developed countries due to overcrediting (compared to the case of the project-by-project baseline) evaluated at the emission price $\mathrm{q}$ (provided that it reflects the externality correctly), and the second term represents the local gain especially due to the savings in the transaction costs, and the third term adjust for the costs of losing exact measure, including risk costs, which we do not model explicitly here. Usually discussion centers around the first term only, and design of mechanism is mostly evaluated if this term is close to 0 . We like to point out that this must be weighed against the effects represented in the rest, especially the loss in environmental value is often canceled out by the increase in the local profits. How one should evaluate this is a major problem posed by this problem.

Obviously, the case here is so simple that there hardly is an informational concern, so that transaction costs can be omitted without doing any harm. Standardization of the baseline has another aspect, which may worsen the world welfare by affecting incentives, when real baseline is not known for sure.

Next assume that $\mathrm{b}$ is distributed according to the distribution function $\mathrm{H}(\mathrm{b})$ while $\mathrm{a}$ is fixed. This situation is close to the one investigated by Millard-Ball (2013) where he assumed that b cannot be observed by the administrator and possibly by the PP. Then under the CDM with the individualized baseline, those projects with $b$ higher than or equal to

$\mathrm{b}(1)=(\mathrm{a}(\mathrm{c} "-\mathrm{d})+\mathrm{K}+\mathrm{F}+\mathrm{ae}) / \mathrm{aq}$

qualify as a CDM project. In this setup, b does not affect the profitability without credits, and therefore additionality constraint does not bite with $\mathrm{F}=\mathrm{F}$ " and $\mathrm{c}$ " $>\mathrm{d}$. This is one extreme situation in that $\mathrm{d}$ is fixed, i.e. hypothetical emission level is totally independent of costs.

Under CDM with a standardized baseline, if

$b^{\prime}>b(2)=(a(c "-d)+F+a e) / q$

then given that $\mathrm{e}<\mathrm{b}$ for all possible values of $\mathrm{b}$ among possible projects, all the projects becomes potentially viable for CDM, but only those projects with latent baseline level $b>b(2)$ would have an incentive to apply for CDM under individualized baseline without transaction costs. If $b^{\prime}<b(2)$, then no project is meritorious. Again the number of CDM projects increase by the chance equaling to $\mathrm{H}(\mathrm{b}(1))-\mathrm{H}(\mathrm{b}(2))$ provided that $b^{\prime}>b(1)$.

This extreme result is due to the fact that $b$ is uncertain but $e$ is certain and also the assumption that high baseline emission level under the alternative scenario is not associated with higher costs, which could be quite unrealistic. As a result, no under-crediting takes place.

For a more general situations, we must consider the case where variation in $b$ is associated with variation in a. Analogous to the previous case, we see that under the individualized baseline, PP has an incentive for CDM if

$\mathrm{K}+\mathrm{F}<\mathrm{a}(\mathrm{c} "-\mathrm{d}+\mathrm{q}(\mathrm{b}-\mathrm{e}))$

and thus low values of a can be compensated by higher value of b, i.e. small firms have incentive when it is relatively dirtier. The opposite holds too, i.e. a lower value of $b$ must be accompanied by relatively higher value of a. That is, for a cleaner firm to have an incentive, it must be large. Under the standardized baseline,

$\mathrm{F}<\mathrm{a}(\mathrm{c}$ '-d+q(b'-e)) 
becomes the condition. As before, given b', only a determines the incentive, provided that $b^{\prime}$ is set sufficiently high.

As a special example, let us examine the case where $a$ and $b$ are perfectly and inversely correlated so that $a b=m$ holds for some $m>0$, i.e. smaller project has some inefficiency in controlling emissions. In this case

$\mathrm{a}^{\prime}(1)=(\mathrm{K}+\mathrm{F}-\mathrm{mq}) /(\mathrm{c} "-\mathrm{d}-\mathrm{eq})$

and

$a^{\prime}(2)=F /\left(c^{\prime \prime}-d+\left(b^{\prime}-e\right) q\right)$

Provided that all the terms in the denominator and the numerator are positive and $\mathrm{K}>\mathrm{mq}$, we have $\mathrm{a}^{\prime}(2)<\mathrm{a}^{\prime}(1)$. Again we have more projects being attracted to CDM by standardization due to saving in transaction costs. However, in this case, initially cleaner projects (i.e. cleaner projects were chosen in the absence of the mechanism) are attracted to under the individualized baseline, and relatively dirtier projects become attractive by standardization. Because of this relationship, over-crediting may take place for projects viable under the individualized baseline, while under-crediting take place for the project only viable under the standardized baseline.

\subsection{Fischer Model}

Fischer (2005) assumed that $\mathrm{b}$ and $\mathrm{e}$ are related by a scale factor, say

$\mathrm{e} / \mathrm{b}=\mathrm{s}$.

If one thinks that the efficiency of management remains the same regardless of the project is qualified as a $\mathrm{CDM}$ or not, then relatively high emission firm remains to be a dirtier firm under CDM. Still we assume that $\mathrm{e}$ is known but does not provide an exact information of $\mathrm{b}$; one may think that s contains a small idiosunclatic risk for each type b. Her analysis includes energy saving revenue with a specific interpretation that the emission reduction is from energy saving, but we adopt her formulation to our setup, here and so additionality does not bite again. If this is the case, then

$$
\mathrm{a}(1-\mathrm{d})-\mathrm{K}-\mathrm{F}+\mathrm{a}(\mathrm{b}-\mathrm{e}) \mathrm{q}=\mathrm{a}(1-\mathrm{d}+(1-\mathrm{s}) \mathrm{bq})-\mathrm{K}-\mathrm{F}
$$

and

$a(1-d)-F+a\left(b^{\prime}-e\right) q=a\left(1-d+\left(b^{\prime}-s b\right) q\right)-F$.

One sees that the variable profit part now is increasing in $b$ under individualized baseline, whereas it is decreasing in $\mathrm{b}$ under standardized baseline. Now the breakeven points for the size parameter are

$a^{*}(1)=(K+F) /(c "-d+(1-s) b q)$

and

$a^{*}(2)=F /(c "-d+(b '-s b) q)$

and which is greater depends upon b' also. When $b^{\prime}$ and $b$ are not so far apart, then still $a^{*}(1)>a^{*}(2)$ holds. However, the gap becomes greater as $b$ increases. Thus the effect of alleviating transaction costs on the number of projects newly attracted to CDM would be greater if baseline or the potential alternative project emission level is higher.

More interesting is the case where $\mathrm{b}$ is uncertain given a. Corresponding breakeven values are

$$
\begin{aligned}
& \mathrm{b}^{*}(1)=\left(\mathrm{K}+\mathrm{F}-\mathrm{a}\left(\mathrm{cc}^{\prime}-\mathrm{d}\right)\right) / \mathrm{a}(1-\mathrm{s}) \mathrm{q} \\
& \mathrm{b}^{*}(2)=\left(\mathrm{a}\left(\mathrm{cc}^{\prime \prime}-\mathrm{d}+\mathrm{b}^{\prime} \mathrm{q}\right)-\mathrm{F}\right) / \mathrm{asq}
\end{aligned}
$$

Now, $b^{*}(2)$ could be negative and on the other extreme, $b^{*}(2)$ could be greater than $b^{*}(1)$. If $b^{*}(2)$ is negative, then no project satisfies incentive condition. If $b^{*}(2)$ is positive, then those projects with $b<b^{*}(2)$ satisfies incentive condition under standardized baseline and so are those projects with $b>b *(1)$ under individualized baseline. This is also noted by some, that standardization favors previously cleaner firms, compared to project-by-project baseline. In this extreme case, adding to this effect, if $0<\mathrm{b}^{*}(2)<\mathrm{b}^{*}(1)$ hold, then the projects attracted to CDM would be completely disjoint from each other (which was the main point discussed in Imai, Akita, and Niizawa (2012)). In this case, all credits are over-crediting with no genuine reduction taking place. 
Imai, Akita, and Niizawa, standardized baseline

Again we may examine the case of perfectly and inversely correlated $a$ and $b$ with $a b=m$. The breakeven points are

$$
\mathrm{a}^{* *}(1)=(\mathrm{K}+\mathrm{F}-(1-\mathrm{s}) \mathrm{mq}) /(\mathrm{c} "-\mathrm{d})
$$

and

$\mathrm{a}^{* *}(2)=(\mathrm{F}+\mathrm{sqm}) /\left(\mathrm{c}{ }^{\prime \prime}-\mathrm{d}+\mathrm{b} ' \mathrm{q}\right)$.

Again the relative magnitude of these two variables would depend upon the value of each parameter, but we may reasonably assume that $\mathrm{K}<\mathrm{mq}$, and then $\mathrm{a}^{* *}(2)<\mathrm{a}^{* *}(1)$ holds as before. Although the reversal as above would not take place, instead, shrinkage of the set of projects attracted to CDM may occur. If the denominator of $\mathrm{a}^{* *}(2)$ is negative, then all projects may be viable under the CDM with a standardized baseline..

\subsection{EB proposal}

EB's proposal at 65 th meeting is to obtain distribution of emission factors from the industry ordered from above (in a descending oreder), and then take four fifth of the distribution as the baseline, i.e. $b$ such that $\mathrm{H}\left(\mathrm{b}^{\prime}\right)=0.2$ holds. Additionality could be that number or higher, e.g. $\mathrm{H}\left(\mathrm{d}^{\prime}\right)=0.1$. However, the real distribution differs from potential distribution, and also ranking by emission factor may not reflect cost consideration, as they may not be perfectly correlated. Furthermore, the real obstacle would be the issue that who bears the costs of collecting such data.

The setup can be given by the situation where c" and b are uncertain (while e and $\mathrm{d}$ are known with a known distribution and perfect correlation between $b$ and $c "$, via $c "=f(b)$. Under the hypothesis of a hidden information, the rest remains the same. That is the proponents of a project know $b$ and $c$ " $=f(b)$, which administrator does not know. Additionality condition (when $F=F$ ") is $1-f(b)>1-d$ and so some initially dirtier projects are excluded. Under the individualized baseline, the benefit becomes

$\mathrm{a}(1-\mathrm{f}(\mathrm{b}))-\mathrm{K}+\mathrm{aq}(\mathrm{b}-\mathrm{e})$

And under the standardized baseline

$\mathrm{a}(1-\mathrm{f}(\mathrm{b}))+\mathrm{aq}\left(\mathrm{b}^{\prime}-\mathrm{e}\right)$. As an example, one may set $\mathrm{f}(\mathrm{b} 9=\mathrm{C}-\mathrm{kb}$, then thecritical values of b's are

$\mathrm{B}(0)=(\mathrm{C}-\mathrm{d}) / \mathrm{k}$

$\mathrm{B}(1)=\{\mathrm{a}(\mathrm{C}-\mathrm{d})+\mathrm{qe})+\mathrm{K}\} / \mathrm{a}(\mathrm{k}+\mathrm{q})$

$\mathrm{B}(2)=\mathrm{a}\left(\mathrm{C}-\mathrm{d}-\mathrm{q}\left(\mathrm{b}^{\prime}\right)\right) / \mathrm{ak}$

If $\mathrm{B}(0)>\mathrm{B}(1)>\mathrm{B}(2)$ holds, then one must beware of the potential addition of projects which did not satisfy the additionalirty condition $(b>B(0))$ due to standardization and hence over-crediting.

Taking advantage of the setups here, one could compare the case where the distribution of $b$ represents the actual alternatives for the project proponents. In this context, a variation of costs is due to difference in technology used. In this case, we should incorporate the aspects of energy saving into the picture. Let $r$ be the price of energy and $t(b)$ be the energy input (expressed in the common unit) necessary for the technology resulting in the emission level $b$. (There could be multiple different technologies yielding the same b like many different renewable energy options, but here we subsume such possibilities for the sake of simplicity.) Then profit maximization implies that

$\mathrm{B}^{*}=\operatorname{argmax}\{\mathrm{a}(1-\mathrm{f}(\mathrm{b})\}-\mathrm{t}(\mathrm{b})\}$.

We could assume that $\mathrm{f}$ is convex and decreasing, while $\mathrm{t}$ is convex and increasing. If we assume the differentiability as well, and assuming the interior maximum, then $0=-f^{\prime}(b)-t^{\prime}(b)$ characterizes the optimum.

Note that the way to locate the baseline emission level this way is quite different from the approach adapted above. From an unobservable list of technologies $\mathrm{f}(\mathrm{b})$, a firm would choose optimal one. Given that this is a private information, for the administrator, possible distribution of $b$ is the one we have discussed in the main text. EB's method uses the real distribution possibly in a certain country as the data to obtain the standardized baseline. 
Imai, Akita, and Niizawa, standardized baseline

One could summarize the relationship among these distributions as follows. Analogous to Fischer's analysis, the last method corresponds to expected and efficient baseline, while individualized baseline corresponds to historical baseline and standardized baseline to industry average baseline. Although all these are admitted in Marrakech Accord, and in this sense the appeal to any of these methods is legitimate, but how to choose among these are not specified. In practice, each methodology announces which category the particular methodology belongs to, and project proponents choose them through their choice of the methodology. Fischer analysed the case where project proponents can choose among these categories which apparently induce some problem like over-crediting. Sometimes it is understood that if the project is the brownfield project, then historical baseline is appropriate, while if the project is greenfield but there are incumbent firm or similar industry exists in its vicinity, then industry average baseline would do, and when there is no reasonable precedence, one must appeal to expected efficient baseline.

For the case raised by EB, we must say that one does not have to standardize the projects in the industries where existing technology varies so much, like power plants.

\section{TENTATIVE CONCLUSION}

The problem of standardization contains several problems. We have reviewed several ones utilizing a simplistic model. This model is so simple that there are apparent extensions desirable, which is one of the future agenda.

We have not stressed the beneficial aspect of standardization that save transaction costs much. In fact, some of the newly attracted projects are those projects not viable because of the transaction costs. owing to addition of those projects, cost efficiencies of emission reduction scheme could be improved. The main issue would be who bear these measurement costs. Even under the current system, use of industry average is allowed as the Marrakech Accord suggests (although actually not many methodologies claim themselves as employing industry averaging), and also it could coexist with the methodology utilizing the historical baseline. However, the current practice requires that individual project proponents must bear the costs of gathering data and also each applicant must duplicate the effort when they utilize the same methodology. Allowing followers to utilize the same data developed by the early applicant may be an option but creates an apparent free-rider problem. The current proposal suggests the local authority conducts the survey to get the data, but if they can afford the costs is still the question. Issues of standardization could be better captured as the issue of avoiding the measurement costs.

\section{ACKNOWLEDGMENTS}

This work was supported by JSPS KAKENHI Grant Number 23310032

\section{REFERENCES}

Fischer (2005) "Project-based Mechanisms for Emissions Reductions: balancing tradeoffs with baselines", Energy Policy, 33, 1807-1823

Hayashi and Michelowa (2012) "Standardization of baseline and additionality determination under the CDM", Climate Policy, iFirst Research Article 1-19

Imai, Akita, and Niizawa (2012) "Theoretical Analysis of Economic Mechanisms In International Environmental Agreement” Conference Proceeding of SGEM2012, Vol 4, 1199-2006

Millard-Ball (2013) "The Troble with Voluntary Emission Trading: Uncertainty and Adverse Selection in Sectoral Crediting Programs,” Journal of Environmental Economics and Management, 65, pp 40-55.

Wara and Victor, (2008) “A Realistic Policy on International Carbon Offsets” Working Paper \#74. April, Stanford: Stanford University, Program on Energy and Sustainable Development. http://iisdb.stanford.edu/pubs/22157/WP74_final_final.pdf 